\title{
Computer-Aided Patient-Specific Coronary Artery Graft Design Improvements Using CFD Coupled Shape Optimizer
}

\author{
Onur Dur, ${ }^{1}$ Sinan Tolga Coskun, ${ }^{2}$ Kasim Oguz Coskun, ${ }^{3}$ David Frakes, ${ }^{4}$ \\ Levent Burak Kara, ${ }^{5}$ and Kerem Pekkan ${ }^{1,5}$ \\ ${ }^{1}$ Department of Biomedical Engineering, Carnegie Mellon University, Pittsburgh, PA, USA; ${ }^{2}$ Department of Vascular Surgery, \\ Horst Schmidt Kliniken, Wiesbaden, Germany; ${ }^{3}$ Department of Thoracic Cardiovascular Surgery, University of Göttingen, \\ Göttingen, Germany; ${ }^{4}$ School of Biological and Health Systems Engineering, Arizona State University, \\ Tempe, AZ, USA; and ${ }^{5}$ Department of Mechanical Engineering, Carnegie Mellon University, \\ Pittsburgh, PA, USA
}

(Received 29 June 2010; accepted 1 November 2010; published online 18 November 2010)

Associate Editor Peter McHugh oversaw the review of this article.

\begin{abstract}
This study aims to (i) demonstrate the efficacy of a new surgical planning framework for complex cardiovascular reconstructions, (ii) develop a computational fluid dynamics (CFD) coupled multi-dimensional shape optimization method to aid patient-specific coronary artery by-pass graft (CABG) design and, (iii) compare the hemodynamic efficiency of the sequential $\mathrm{CABG}$, i.e., raising a daughter parallel branch from the parent CABG in patient-specific 3D settings. Hemodynamic efficiency of patient-specific complete revascularization scenarios for right coronary artery (RCA), left anterior descending artery (LAD), and left circumflex artery (LCX) bypasses were investigated in comparison to the stenosis condition. Multivariate 2D constraint optimization was applied on the left internal mammary artery (LIMA) graft, which was parameterized based on actual surgical settings extracted from 2D CT slices. The objective function was set to minimize the local variation of wall shear stress (WSS) and other hemodynamic indices (energy dissipation, flow deviation angle, average WSS, and vorticity) that correlate with performance of the graft and risk of re-stenosis at the anastomosis zone. Once the optimized 2D graft shape was obtained, it was translated to 3D using an in-house "sketch-based" interactive anatomical editing tool. The final graft design was evaluated using an experimentally validated second-order non-Newtonian CFD solver incorporating resistance based outlet boundary conditions. 3D patient-specific simulations for the healthy coronary anatomy produced realistic coronary flows. All revascularization techniques restored coronary perfusions to the healthy baseline. Multi-scale evaluation of the optimized LIMA graft enabled significant wall shear stress gradient (WSSG) relief $(\sim 34 \%)$. In comparison to original LIMA graft, sequential graft also lowered the WSSG by $15 \%$ proximal to LAD and diagonal bifurcation. The proposed sketchbased surgical planning paradigm evaluated the selected
\end{abstract}

Address correspondence to Kerem Pekkan, Department of Biomedical Engineering, Carnegie Mellon University, 700 Technology Dr., Pittsburgh, PA 15219, USA. Electronic mail: kpekkan@ andrew.cmu.edu coronary bypass surgery procedures based on acute hemodynamic readjustments of aorta-CA flow. This methodology may provide a rational to aid surgical decision making in time-critical, patient-specific CA bypass operations before in vivo execution.

Keywords-Surgical planning, Coronary artery, Bypass graft, CFD, Hemodynamics, Shape optimization, Sequential graft, WSS, WSSG, Surgical design.

\section{INTRODUCTION}

Statistics from the American Heart Association identify coronary heart diseases (CHD) as the principal cause of morbidity and mortality in the western world. ${ }^{14,42}$ Major causes of CHD include atherosclerosis and complications related to congenital cardiac defects. Atherosclerosis involves the agglomeration of fatty substances, cholesterol, and other deposits on the inner lining of an artery together with transverse growth of smooth muscle cells (i.e., artheroma). This results in reduced blood flow and other pathological complications. $^{42}$

Bypass conduits provide an alternative route around critically blocked arteries. Current surgical anastomosis techniques and the design of synthetic coronary artery bypass grafts $(\mathrm{CABG})$ frequently lead to post-surgical complications such as intimal thickening, restenosis, and eventual long term graft failure. Failure presents in $5-20 \%$ of patients within $1-5$ years, and approximately $50 \%$ of patients within 10 years after CABG surgery. ${ }^{5}$ Pathological hemodynamic states are usually precursors of intimal hyperplasia or platelet deposition and may result in graft occlusion. $^{6,13,57}$ From a fluid mechanics perspective, 
abnormalities in coronary flow include recirculation zones, low/oscillating shear stresses, vortices, and areas of stagnation within the CABG. These parameters relate to the variation in strain rate within the conduit, which in turn are influenced strongly by the shape of the flow domain. ${ }^{28}$ Therefore, in order to improve the success of the surgery, the optimal anastomosis geometry and angle have been actively researched. Walsh et al. ${ }^{53}$ demonstrated that the use of cuffs (i.e., Miller cuff) and patches (i.e., Taylor patch) can significantly reduce abnormal wall shear stress (WSS) and wall shear stress gradient (WSSG) by up to $60 \%$ in patient-specific models, when compared to a conventional distal end-to-side anastomosis. Studies based on idealized femoral bypass geometries provided a better insight on the influence of various design parameters such as the advantage of an acute anastomosis angle, i.e., $10-20^{\circ}, 3,11$ creating enlarged lumen sections around the toe region to reduce WSS parameters and enable smooth transition of the flow from graft to host artery, ${ }^{3,22,23}$ and influence of the proximal artery flow. ${ }^{3,20}$ Apart from these local design considerations, the bulk shape of the bypass conduits has received little attention. Based on the rapid variation of high and low wall shear stress along the sinusoidal shaped vessel geometries, ill-shaped grafts may also be prone to atherosclerosis development.

Computational fluid dynamics (CFD) provide a viable tool for pre-surgical planning and device design, and for improving the design of surgeries and interventions used in cardiovascular medicine..$^{33,35,40,49-51}$ Coupled with accurate reconstructions of anatomical data (via magnetic resonance imaging, angiograms, or computational tomography), ${ }^{12} \mathrm{CFD}$ simulations provide the ability to quantify local hemodynamics and allow evaluating the performance of surgical design templates ${ }^{26,27,37}$ and candidate endovascular devices. ${ }^{58}$

Anatomical three-dimensional shape editing is one of the major challenges of the pre-surgical planning paradigm as cardiovascular geometries involve nonuniform vessel caliber and curvature, and conduits require complex multiple inlet-outlet geometries, which cannot be easily modified by combinations of mathematically simple binary operations or shape primitives offered by state-of-art CAD software. ${ }^{19} \mathrm{We}$ introduced the first generation 'interactive' surgical planning tool, SURGEM,${ }^{36}$ which incorporates a twohand haptic interface to freely deform, bend and position 3D surgical baffles real-time. This shapemorphing tool has been used successfully in routine basis to aid the pre-surgical decision making process of congenital heart surgeries before the in vivo execution. $^{37,49}$ Integration of a sketch-based 3D platform $^{16-18}$ now expands the capability of 'interactive' anatomy-editing systems by replacing the expensive haptic user interface with an easy-to-access digital sketch-based modeling environment, i.e., tablet, that takes user strokes as input, and seamlessly converts them into precise three-dimensional (3D) geometric data. This allows surgeons to construct and edit anatomical structures directly in 3D precisely the way they envisage on a two dimensional (2D) image.

To date, CFD has been utilized primarily for identifying an optimal design, based on trial-and-error, among a small number of geometrical variations and intuitive design alternatives. More recently, several investigators have demonstrated the benefits of coupling computer simulations with numerical shape optimization to provide cost-effective methods for the design of the medical devices, ${ }^{1,15}$ surgical connections, ${ }^{25}$ and particularly CABGs. ${ }^{2,39,43}$ The challenges associated with CFD coupled shape optimization for clinical problems have been identified previously by Marsden et al. ${ }^{25}$

Current CABG design paradigms target improved hemodynamics to achieve reduced hyperplasia at the distal anastomosis region by modulating the anastomosis angle ${ }^{1}$ and vessel curvature ${ }^{39,43}$ in simplified 2D tubular conduits. Studies incorporating out-of-plane features reported notable variations in end-to-side anastomosis hemodynamics. ${ }^{31,47}$ Hence, although in-plane (2D) optimization is appropriate to identify the primary design features, an accurate assessment on the CABG hemodynamics requires patient-specific 3D anatomical information for reliable feedback.

One objective of this study was to demonstrate the efficacy of a novel sketch-based surgical planning framework for complex cardiovascular problems, and to develop a CFD coupled multi-dimensional shape optimization method to aid patient-specific CABG design. Hemodynamic efficiency of patient-specific complete revascularization scenarios for right coronary artery (RCA), left anterior descending artery (LAD) and left circumflex artery (LCX) bypasses were investigated in comparison to the stenosis condition. Single objective multivariate constraint optimization was applied to improve the patient-specific design of the end-to-side anastomosis of left internal mammary artery (LIMA) to the distal site of the stenosed LAD. The 2D optimization procedure was comprised of geometry creation, parameterization, mesh generation, finite element (FEA) solution, and design optimization. Multiple hemodynamic indices including the local gradient of WSS, space-averaged WSS, energy efficiency, and recirculation at the anastomosis zone were selected as cost functions for the optimization problem. The optimized 2D graft shape was re-evaluated in a patient-specific 3D setting to validate the efficacy of the 2D optimization methodology. In addition, hemodynamic efficiency of the sequential grafting strategy was 
analyzed in comparison to the standard single $\mathrm{CABG}$ configuration. Sequential grafting is a routine surgical method of raising multiple braches from a parent bypass graft when atherosclerotic occlusions involve more than one coronary artery (CA). Clinically, local hemodynamic adjustments after sequential grafts remain unclear, and there is no established preference for single or sequential graft bypass. ${ }^{41}$

\section{METHODS}

\section{Reconstruction of Patient Anatomy}

Patient-specific anatomy of a 54-year-old patient with CHD was acquired from post-op computerized tomography (CT) images as shown in Fig. 1. The patient underwent a conventional coronary bypass operation to restore the myocardial perfusion through three stenosed major coronary arteries; RCA, LAD, and LCX. End-to-side anastomosis of two saphenous vein grafts and LIMA graft, which is the gold standard based on the higher potency rate, ${ }^{41}$ were performed on RCA, LCX, and LAD, respectively. CT images with $20 \mathrm{lp} / \mathrm{cm}(\sim 0.25 \mathrm{~mm})$ in-plane resolution and $0.5 \mathrm{~mm}$ spacing (400 2D slices) were pre-processed using a free DICOM viwer, DicomWorks (Lyon, France) to optimize image contrast. Aorta-coronary geometry (excluding the grafts) was reconstructed using Simpleware-Scan IP (Simpleware Ltd., Innovation Centre, Exeter, UK). 3D volumetric reconstruction of
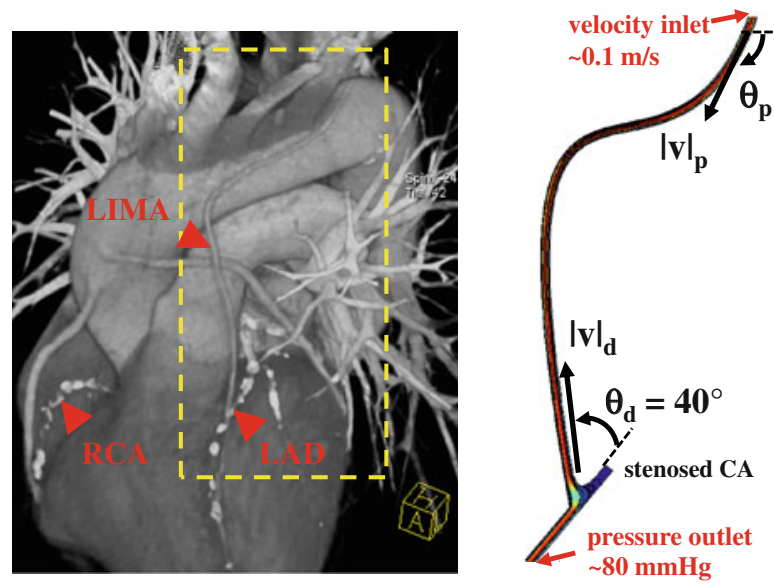

FIGURE 1. Post-operative CT scan of the complete revascularization procedure (left). Right coronary artery (RCA), left anterior descending artery (LAD), and left internal mammary artery (LIMA) are marked with arrows. The area inside the dashed lines indicates the anatomical section of 2D shape optimization. In-plane geometrical features of the LIMA graft were extracted from the CT image (right). In-plane optimization of the LIMA graft model was based on four design parameters; the proximal $\left(\theta_{\mathrm{p}}\right)$ and distal $\left(\theta_{\mathrm{d}}\right)$ anastomosis angles, proximal $\left(v_{\mathrm{p}}\right)$ and distal curvature vectors $\left(v_{\mathrm{d}}\right)$. Inlet and outlet boundary conditions are labeled on the 2D model plotted on the left. the aorta-coronary model was refined in Geomagic (Geomagic Inc., NC, USA) to improve the surface grid resolution. This protocol was successfully demonstrated in earlier studies on complex cardiovascular anatomies. ${ }^{12,34,55}$

To demonstrate the efficacy of our in-house anatomical editing tool, 3D CABGs (LAD, LCX, LIMA, and sequential grafts) were created virtually based on the post-op CT patient data. This interactive surgical planning platform is based on the robust shapemorphing principles introduced earlier, ${ }^{36}$ and incorporates B-spine curves for generating highly flexible tubular conduits. The sketch-based computer interface allows surgeon/operator to use pen strokes to modulate the in-plane design features (i.e., curvature, proximal, and distal anastomosis points) of the 3D tubular conduit, while preserving all out-of-plane features that reside on the remaining planes. Hence, rotating the view plane surgeon can switch between on multiple design planes and perform the desired 3D shape adjustments. This 'iterative multi-plane shape-design' approach allows generating realistic bypass grafts virtually under 10 min of user time.

\section{CFD Coupled 2D Shape Optimization}

The two-dimensional (2D) optimization framework incorporated a CFD-based evaluation of the cost function into the optimization algorithm in an automated fashion (Fig. 2). User defined subroutines were used to generate the desired $\mathrm{CABG}$ shape variations

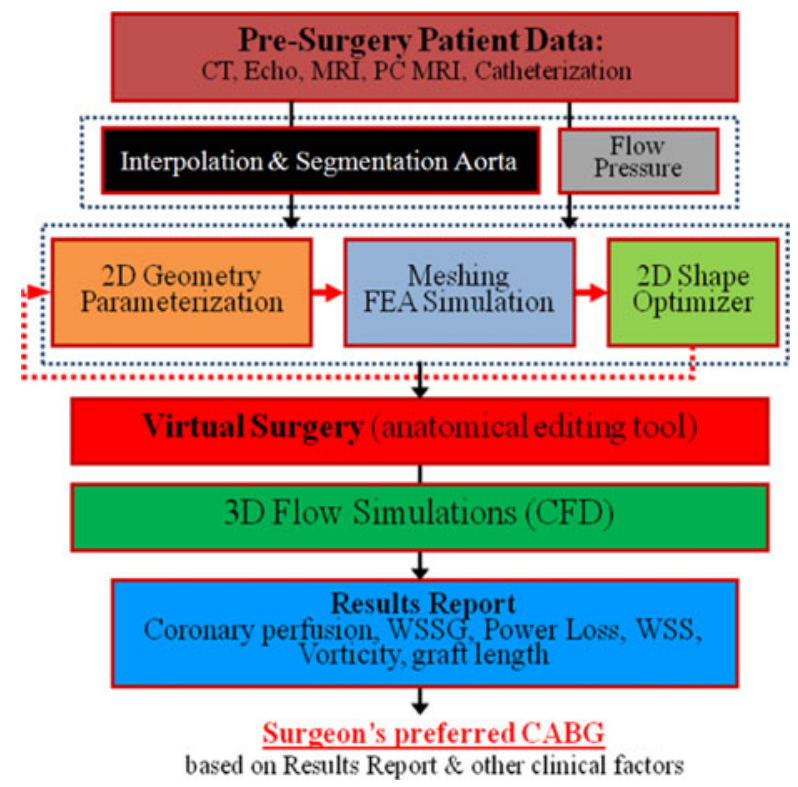

FIGURE 2. Flowchart of the proposed pre-surgical planning framework. This workflow enables cyber-generated intelligence to aid surgical decision making for patient-specific CABG procedures. 
and to allow a robust communication between the Optimization Toolbox of MATLAB (Mathworks Inc., Natick, MA) and the FEA solver of FEMLAB (COMSOL Inc., Burlington, MA).

The optimization procedure was applied to the LIMA graft, which was parameterized based on actual surgical settings extracted from static 2D CT images as shown in Fig. 1. The CABG was simplified as a 2D cylindrical tube for the in-plane optimization. The scaffold of the coronary vessel was based on a thirdorder Bezier curve whose shape was dictated by four design parameters $(s)$ : the proximal $\left(\theta_{\mathrm{p}}\right)$, and distal $\left(\theta_{\mathrm{d}}\right)$ anastomosis angles and proximal $\left(v_{\mathrm{p}}\right)$ and distal curvature vectors $\left(v_{\mathrm{d}}\right)$. The latter two parameters defined the curvature of the conduit away from the anastomosis regions. The final 2D axisymmetric tube was formed by extruding the vessel walls in the normal directions along the centerline. In order to consider the hemodynamics of the proximal anastomosis zone, the LAD artery was also included in the optimization model as a static 2D cylindrical tube with corresponding patient-specific vessel caliber and orientation based on the CT image. Second-order Bezier curves were used to smooth geometric perturbations and sharp-corners at the anastomosis zone.

The 2D geometry of the anastomosis region was discretized using unstructured tetragonal mesh that enabled high mesh quality for dynamic shape alterations during optimization. Grid independence was ensured by comparing solutions at six refinement levels. The results presented in this manuscript were based on 9k triangular elements. In addition, local grid sensitivity checks were performed in order to guarantee grid quality proximal to the anastomosis zone. A steady state solver using the Petrov-Galerkin finite-element formulation was incorporated to solve the governing Navier-Stokes $(\mathrm{N}-\mathrm{S})$ equations for each geometric configuration.

A parabolic velocity profile $\left(V(s)=V_{\max } \cdot s(1-s)\right)$ where $V_{\max }$ is the maximum velocity in the core region and $s$ is the arch length along the inlet boundary, was prescribed at the inlet boundary of the graft to yield the typical coronary flow velocity $(0.1 \mathrm{~m} / \mathrm{s}) .{ }^{10}$ No slip boundary condition was enforced on the vessel walls and the proximal end of the LAD, which was assumed to be completely $(100 \%)$ stenosed. No traction boundary condition was assigned at the distal end of LAD with a constant myocardial pressure of $10 \mathrm{mmHg}$. In order to consider the non-Newtonian behavior of coronary blood in low shear regions, two blood rheology models (Ballyk and Carreau ${ }^{48}$ ) were implemented. Blood density was specified as $1060 \mathrm{~kg} / \mathrm{m}^{3}$. Simulations were performed on a dualcore T7200 computer with $2 \mathrm{~GB}$ of memory. Approximately $5 \mathrm{~min}$ were required to complete one converged steady state solution.

\section{Optimization Problem and Cost Functions}

The single objective multivariate constraint optimization was performed using the Sequential Quadratic Programming (SQP) method. SQP provides efficient and accurate evaluation of constrained optimization routines $^{46}$ by attempting to solve for the Lagrange multiplier $(\lambda)$ directly. The search direction to achieve the global minima is provided by formulating a quadratic sub-problem based on a quadratic approximation of the Lagrangian, $L(s, \lambda)$

$$
L(s, \lambda)=F(\vec{U}(s), s)+\sum_{i=1}^{m} \lambda_{i} g_{i}(s)
$$

In the above formula, $\vec{U}(s)$ and $F(\vec{U}(s), s)$ refer to the flow solution and cost function evaluation, respectively. The bulk shape of the LIMA graft and distal anastomosis region was controlled using three $(m=3)$ of the four design parameters, $s: \theta_{\mathrm{d}}, v_{\mathrm{p}}$, and $v_{\mathrm{d}}\left(\theta_{\mathrm{p}}\right.$ is fixed). Major anatomical constraints due to pulmonary vasculature, aorta, and heart and lungs were extrapolated from the 2D CT image (Fig. 1, the design plane) and incorporated in the optimization. Inequality constrains $\left(g_{i}\right)$, upper $\left(s_{\mathrm{u}}\right)$, and lower $\left(s_{1}\right)$ bounds for each design parameter were based on the 2D anatomical constraints such that the altered LIMA graft geometry do not overlap with (i) the head-neck vessels of aorta at the proximal anastomosis, (ii) myocardial tissue on the left side of the distal anastomosis, and (iii) with the proximal pulmonary vasculature on the right. This allowed realistic geometric variations with no overlapping sections, i.e., to avoid singularity in shape. To converge upon the global minimum, initial conditions for all the design variables were altered within the allowable range set by the inequality constrains. Details of the optimization problem are formulated below.

$\min _{S \in \Re^{n}} F(\vec{U}(s), s)$

where:

$\vec{U}=\left|\begin{array}{l}\vec{u}(s) \\ P(s)\end{array}\right|$ (state variables), $\quad s$ (design parameters)

$s: v_{\mathrm{d}}, v_{\mathrm{p}}, \theta_{\mathrm{d}}, \theta_{\mathrm{p}}$

subject to:

$$
\begin{aligned}
& \rho \frac{D \vec{u}}{D t}=-\nabla P+\nabla \cdot \eta\left(\nabla \vec{u}+\nabla \vec{u}^{\mathrm{T}}\right) \quad \nabla \cdot \vec{u}=0 \\
& g_{i}(s) \leq 0, \quad i=1,2, \ldots, m \\
& s_{1} \leq s \leq s_{\mathrm{u}}
\end{aligned}
$$

Based on both computational simulations and clinical observations as in Lei et $a .^{23}$ local spatial variation of wall shear stress acts as a strong 
TABLE 1. Summary of hemodynamic cost functions evaluated for CFD-coupled 2D CABG optimization.

\begin{tabular}{|c|c|}
\hline Cost function & Formulation \\
\hline $\begin{array}{l}\text { Velocity gradient } \\
\text { based energy } \\
\text { dissipation }\end{array}$ & $E_{\mathrm{loss}}=\frac{1}{V} \int \phi d v, \quad \phi=\frac{1}{2} \mu\left(\frac{\partial u_{i}}{\partial X_{j}}+\frac{\partial u_{j}}{\partial X_{i}}\right)$ \\
\hline Severity parameter & $\begin{array}{l}\mathrm{SP}=\frac{1}{A} \int \mathrm{WSSG} d s, \\
\mathrm{WSSG}=\sqrt{\left(\frac{\partial W S S}{\partial x}\right)^{2}\left(\frac{\partial W S S}{\partial y}\right)^{2}\left(\frac{\partial W S S}{\partial z}\right)^{2}}\end{array}$ \\
\hline $\begin{array}{l}\text { Pressure drop } \\
\text { Mean wall shear } \\
\text { stress }\end{array}$ & $\begin{array}{l}\Delta P=P_{\text {in }}-P_{\text {out }} \\
\mathrm{WSS}_{\mathrm{m}}=\frac{1}{A} \int \mathrm{WSS} d s, \mathrm{WSS}=\mu\left(\Delta u+(\Delta u)^{\top}\right)\end{array}$ \\
\hline $\begin{array}{l}\text { Flow deviation angle } \\
\text { Vorticity }\end{array}$ & $\begin{array}{l}\phi=\frac{1}{V} \int|\operatorname{dev}| d V, \quad \operatorname{dev}=a \tan \left(u_{\mathrm{N}-\mathrm{S}} / u_{\mathrm{S}}\right) \\
\zeta^{2}=\frac{1}{V} \int\left|\omega^{2}\right| d V, \quad \omega=\vec{\nabla} \times \vec{u}\end{array}$ \\
\hline
\end{tabular}

Severity parameter and WSS are integrated along the boundary surfaces $(d s)$ and normalized with total area $(A)$. Energy loss, flow deviation angle, and vorticity are space-averaged over the volume (V). N-S and S refer to the Navier-Stokes and Stokes flow solutions.

determinant of myointimal hyperplasia. In our study, severity parameter (SP), which quantifies the average wall shear stress gradient at the anastomosis zone, was chosen as the cost function, i.e., $F(\vec{U}(s), s)$, for the optimization problem. As summarized in Table 1, additional cost functions including the energy loss $\left(E_{\text {diss }}\right)$ and pressure drop $(\Delta P)$ along the LIMA graft, the average WSS $(\overline{\mathrm{WSS}})$, vorticity $\zeta$, and flow deviation angle $(\Phi)$ at the anastomosis zone were also evaluated. $E_{\text {diss }}$ and $\Delta P$ correlates with the energy efficiency of the surgical conduit and have been used to quantify the performance of the complex reconstructive surgical connections. ${ }^{34,44} \overline{\mathrm{WSS}}$ is commonly recognized as a major mechanical determinant of vasoregulation and disease state. ${ }^{24} \zeta$ and $\Phi$ are the measures of circulation and rotation in the fluid, thus they highlight the regions with recirculation or turbulence, which may cause pathologic vascular response and blood trauma. ${ }^{15} \Phi$ was calculated by integrating the angle between the flow vectors evaluated by the $\mathrm{N}-\mathrm{S}$ solution and Stokes solution at the anastomosis zone.

\section{D Hemodynamic Evaluation}

Once the optimized 2D graft shape was obtained, it was translated to $3 \mathrm{D}$ environment by using the in-house anatomical editing tool. This procedure involved three steps: (i) construction of the 2D scaffold of the optimal graft geometry on the $2 \mathrm{D}$ design plane (overlaying the 3D aorta-coronary geometry) based on four design parameters identified by the optimization, (ii) modulation of the 2D geometry on multiple sketch planes to maintain a realistic 3D curvature while preserving the design parameters at

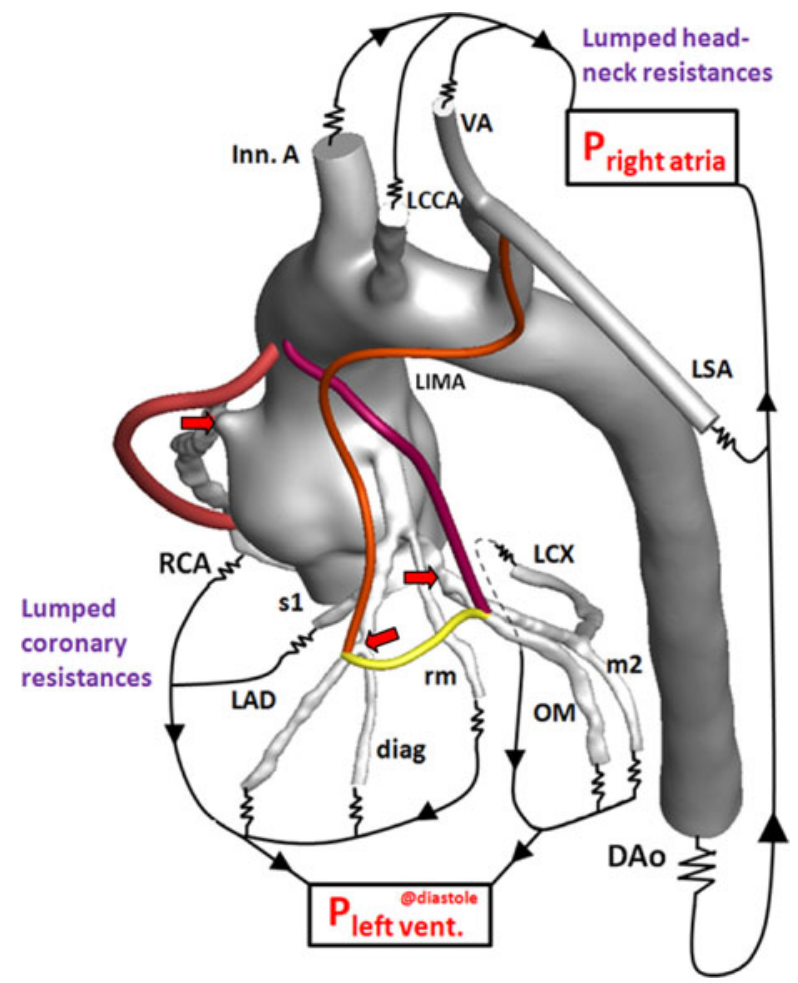

FIGURE 3. Patient-specific 3D aorta-coronary artery model incorporated resistance outlet boundary conditions. Coronary and systemic circulation circuits were closed with the left ventricle and right atrial pressure in diastole, respectively. The aorta-coronary anatomy is comprised of right coronary artery (RCA), left circumflex artery (LCX), left anterior descending artery (LAD), obtuse marginal (OM), second marginal artery (m2), first septal artery (s1), diagonal artery (diag), ramus marginalis ( $\mathrm{rm})$, left internal mammary artery (LIMA), descending aorta (DAo), Innominate artery (Inn. A), left common carotid artery (LCCA), vertebral artery (VA), left subclavian artery (LSA). RCA (red), LIMA (orange), LCX (pink), and sequential grafts (yellow) were displayed color coded. Stenosis locations were marked with red colored arrows.

the original 2D design plane, (iii) generation of the 3D annular graft geometry based on constant diameter surface lofting along the scaffold. Efficiency of the final graft design was evaluated using the experimentally validated second-order solver of Fluent (ANSYS Inc., PA) incorporating resistance boundary conditions that ensures realistic flow distribution along the coronary artery tree (Fig. 3). This solver was originally developed for complex subject-specific anatomical flows. ${ }^{9,35,54,55}$ The 3D flow domain was discretized using $\sim 2$ million unstructured tetrahedral elements using Gambit 2.3.6 (ANSYS Inc., PA). To address the numerical stability problems due to the high systemic resistances of multi-outlet aorta-coronary anatomy (i.e., since even the minute flow rate adjustments are transferred as large pressure oscillations in 3D domain), an iterative under-relaxation-based resistance boundary condition was coupled to each outlet. At each inner iteration, the pressure gradient 


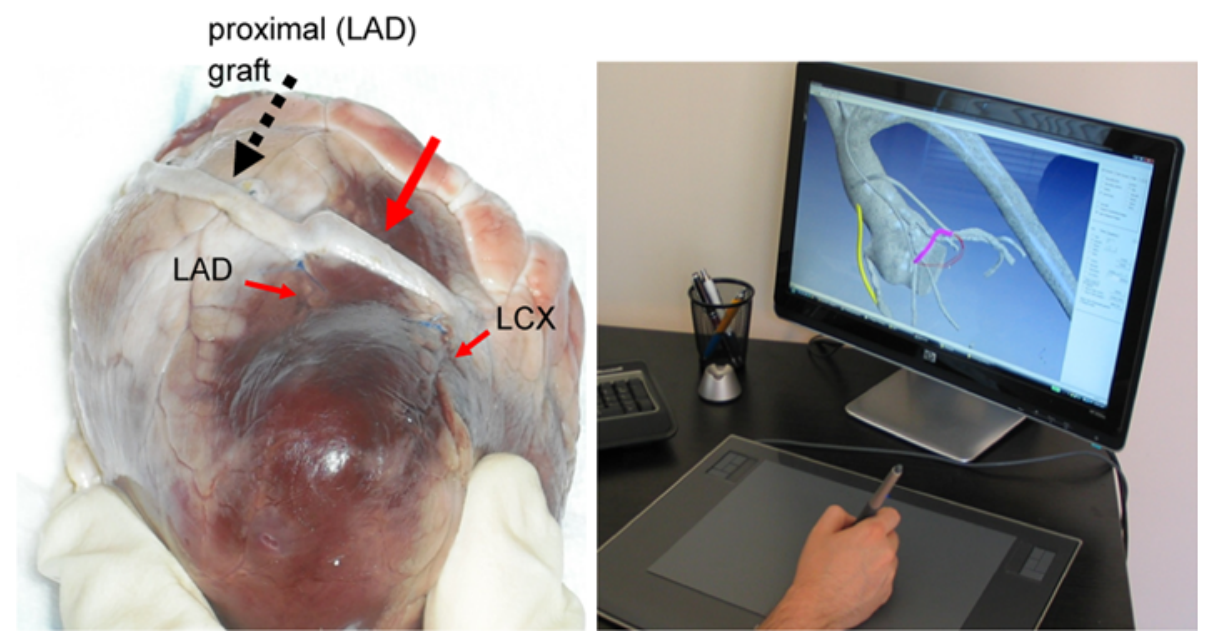

FIGURE 4. A sequential graft (thick solid arrow) between the left anterior descending artery (LAD) and left circumflex artery (LCX) was created by mimicking the actions of the surgeon on a bovine heart template in the operating room (left) using the sketch-based in-house anatomical editing tool (right). Geometry of the 3D sequential graft geometry (pink colored conduit [right]) was adjusted based on pen-strokes of the operator on a sketch-based computer interface.

calculated at the outlets was attenuated ten times to ensure smoother convergence. Each resistance was coupled to the solver iteratively to prevent divergence due multiple outlets. Vessel lumen area was constricted by $50 \%$ proximal to all RCA, LAD, and LCX in order to model the typical moderate stenosis case. Aforementioned non-Newtonian (shear-thinning) rheology models were compared with reference Newtonian model based on non-Newtonian importance factor, $I_{\mathrm{L}}^{48}$ as shown below.

$$
I_{\mathrm{L}}=\frac{\mu}{\mu_{\infty}}, \quad \text { where } \mu_{\infty}=0.00345 \mathrm{~Pa} \mathrm{~s}
$$

Alternative to the single LAD and LCX bypasses, a sequential graft between the LAD and LCX was created by mimicking the actions of the surgeon on a template bovine heart, using the in-house anatomical editing tool as shown in Fig. 4. Geometry of the 3D sequential graft geometry was adjusted based on penstrokes of the operator on a sketch-based computer interface. Hemodynamic efficiency of the LAD-LCX sequential graft was evaluated in $3 \mathrm{D}$ patient-specifics settings. 3D WSS, WSSG, and pressure fields were analyzed in detailed. Error spans reported for WSS calculations were based on one standard deviation with $85 \%$ confidence interval.

\section{RESULTS}

\section{Shape Optimization of the 2D LIMA Graft}

Fully automated coupling between optimal shape design and 2D non-Newtonian blood flow simulations altered the CABG geometry through non-intuitive
LIMA graft alternatives to minimize the severity parameter as shown in Fig. 5. Convergence was achieved within 100 to 400 iterations depending on the initial condition and the cost function. Convergence to global minima depended strongly on the initial condition (i.e. up to $\sim 10 \%$ variation in cost function evaluation due to different initial condition) validating our approach to use multiple initial conditions. For the optimized 2D LIMA graft design, severity parameter was found to be $58 \%$ less than the initial value that was evaluated at the in vivo orientation of the patientspecific LIMA graft. Table 2 shows that $\Delta P$ and length of the graft was decreased about $10 \%$, whereas, $\overline{\mathrm{WSS}}$, $\Phi$, and $E_{\text {diss }}$ remained approximately constant. Flow decelerated considerably $(\sim 40 \%)$ at the anastomosis region with the enlargement of vessel caliber and generated static recirculation zones close to the proximal end of the stenosed LAD. Optimization with respect to energy efficiency related cost functions, i.e.,

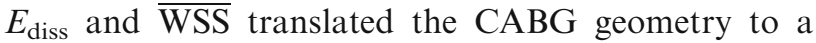
lower curvature shape with reduced cord length. Similarly, optimization with respect to recirculation parameter, $\Phi$ yielded a relatively slack shape by minimizing the magnitude of the curvature vectors. Convergence rate and stability of the solutions were considerably slower due to the low gradient variation of $\Phi$ between intermediate iterations.

\section{Evaluation of 3D Patient-Specific CABG Configurations}

Detailed WSS and pressure distribution maps were generated for each CABG configurations as shown in Figs. $6 \mathrm{a}$ and $6 \mathrm{~b}$, respectively. For the healthy baseline case, WSS was moderately high $\left(\sim 60\right.$ dynes $\left./ \mathrm{cm}^{2}\right)$ at the 


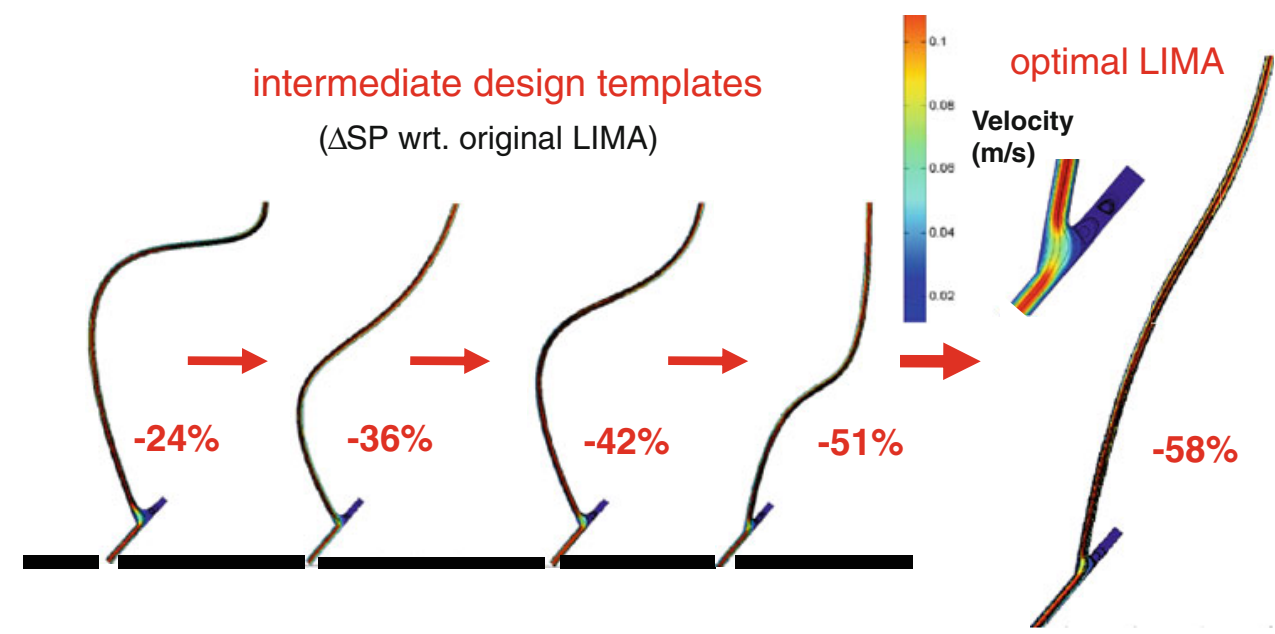

FIGURE 5. Summary of results from the CFD-coupled single objective multivariate 2D optimization for proximal anastomosis. Optimization altered the CABG geometry through non-intuitive LIMA graft alternatives to minimize the average wall shear stress gradient, i.e., severity parameter along the graft and host artery. The final graft shape converged to the minimum curvature state (curvature vectors, $v_{p}$ and $v_{d}$ minimized). Percentages indicate the variation of objective function (severity parameter, SP) between each geometric iteration with respect to the initial configuration.

TABLE 2. Variation of performance parameters between original and optimal LIMA graft configurations based on multivariate single objective, i.e., based on severity parameter (SP), CFD-coupled 2D shape optimization.

\begin{tabular}{lcl}
\hline Original LIMA & \%Variation & \multicolumn{1}{c}{ Optimal LIMA } \\
\hline$E_{\text {diss }}=35.3 \mathrm{~W} / \mathrm{m}^{3}$ & -0.5 & $E_{\text {diss }}=35.1 \mathrm{~W} / \mathrm{m}^{3}$ \\
$\mathrm{SP}=703 \mathrm{~N} / \mathrm{m}^{3}$ & -58 & $\mathrm{SP}=300 \mathrm{~N} / \mathrm{m}^{3}$ \\
$\Delta P=11 \mathrm{mmHg}$ & -12 & $\Delta P=9.6 \mathrm{mmHg}$ \\
$\mathrm{WSS} \mathrm{m}_{\mathrm{m}}=7.2 \mathrm{dyn} / \mathrm{cm}^{2}$ & -1.4 & $\mathrm{WSS} \mathrm{m}=7.1$ \\
$|\Phi|=7.4^{\circ}$ & -4 & $|\Phi|=7.1^{\circ}$ \\
$\zeta^{2}=9448 \mathrm{~s}^{-2}$ & 1 & $\zeta^{2}=9551 \mathrm{~s}^{-2}$ \\
$L=18.2 \mathrm{~cm}$ & -11 & $L=16.2 \mathrm{~cm}$ \\
\hline
\end{tabular}

Significant reductions in $\mathrm{SP}, \Delta P$, and $L$ are achieved by the shape optimization. $E_{\text {diss, }}$, energy dissipation; $\Delta P$, pressure drop between proximal to distal ends of the graft; WSS $_{m}$, mean wall shear stress along the graft walls, $\Phi$ : flow deviation angle, $\zeta$ : vorticity parameter, $\mathrm{L}$ : cord length of the graft.

entrance region of the left main (LM) coronary artery, the downstream curvature of RCA, and proximal to the bifurcation regions. Due to the regression of the vessel caliber, WSS increased gradually along the distal coronary artery tree. After the stenosis was introduced, local WSS was elevated at periphery of the constricted lumen and at opposing wall of the bifurcation downstream of the stenosis due to flow impingement. Compared to the healthy case, average WSS on the left main artery decreased by $22 \%$ after the stenosis and remained at this level for all revascularization scenarios. Space-averaged WSS on the bypass conduits was $6 \pm 2$ dynes $/ \mathrm{cm}^{2}$, which was significantly lower $(\sim 70 \%)$ than the average WSS calculated along the healthy coronary arteries $\left(\sim 22 \pm 4\right.$ dynes $\left./ \mathrm{cm}^{2}\right)$. Elevated WSS zones were localized at the toe region of the distal anastomosis for RCA and sequential grafts, whereas for the LIMA grafts, WSS distribution was uniform and elevated peripherally around the distal anastomosis zone. Addition of the sequential graft between LAD and obtuse marginal lowered the local WSS at the toe region LAD-LIMA anastomosis by $32 \%$ and average WSS along the LAD by $23 \%$. LIMA graft increased the WSS uniformly along LAD (by $13 \%$ ) for both original and optimized LIMA graft designs. After revascularization, WSS on the diagonal artery remained constant (the same level after stenosis) as LIMA graft flow followed solely the anterograde flow path along the LAD without flow reversal toward the proximal bifurcation.

As shown in Table 3, 3D patient-specific simulations for the healthy coronary anatomy produced realistic coronary flows, which agree well with the previous ultrasound angiography measurements. ${ }^{4,56}$ After the moderate stenosis $(50 \%)$ was introduced in the computer, coronary perfusion was reduced notably, by 11,14 , and $24 \%$ along the LAD, LCX, and RCA respectively. In contrast, the post-stenosis coronary outlet pressure was increased only slightly $\sim 1 \mathrm{mmHg}$ (Fig. 6b). Pressure distribution of the healthy case was restored for all revascularization cases but the sequential grafting case, where pressure at proximal sites of the RCA and LAD were slightly higher $\sim 1 \mathrm{mmHg}$. Relative flow variations within the first branches of coronary tree were detailed in Table 3 for each CABG configuration. For $50 \%$ area reduction, all revascularization techniques restored the coronary perfusions to the healthy baseline. LIMA graft shape optimization resulted in negligible variation the coronary perfusion along the arterial tree (less than 2\% change) as the coronary bed vascular impedance was 
(a)

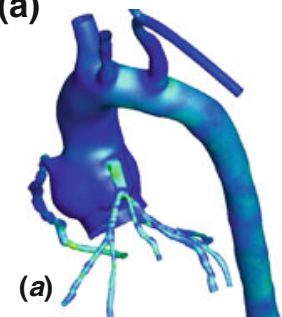

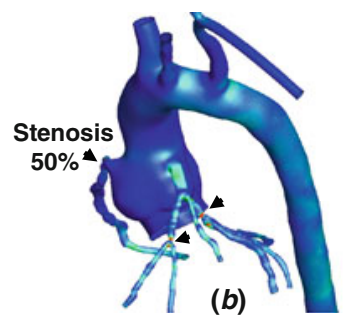

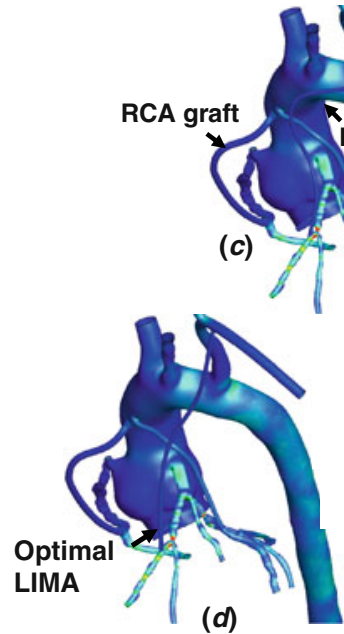

(d)

(b)

(b)
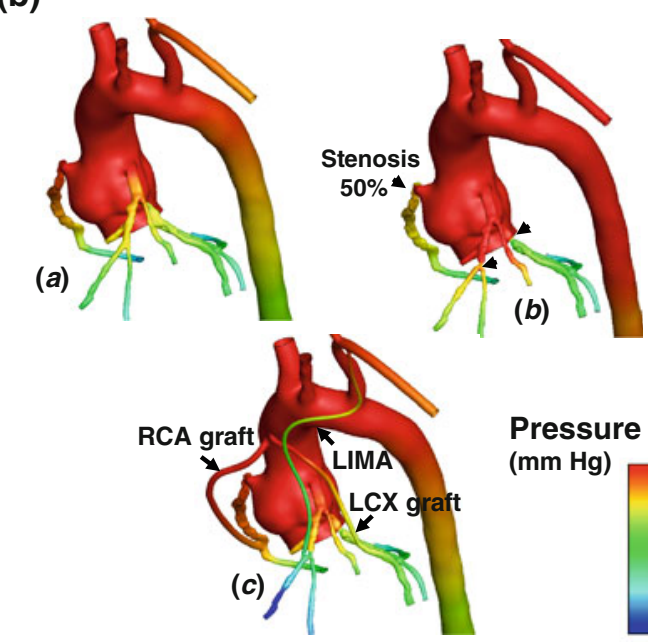

Pressure

(mm Hg)
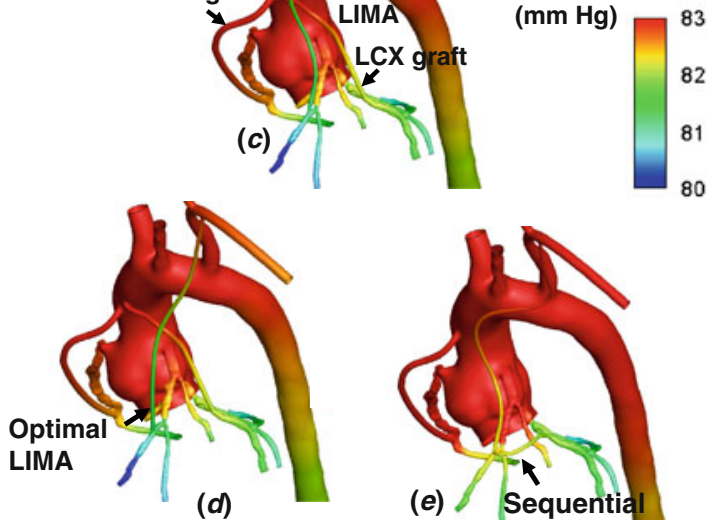

(d)

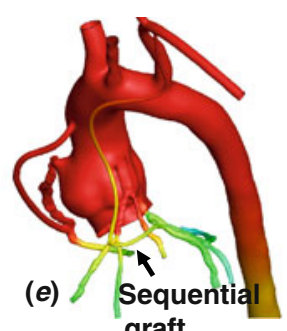

(e) Sequent

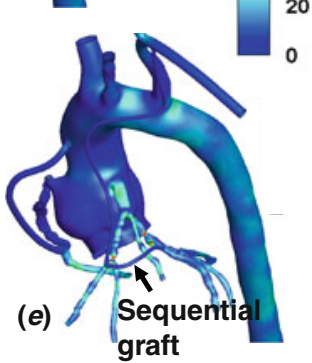

FIGURE 6. (a) Comparison of pressure distribution among the healthy (a), stenosed (b) and single graft revascularization (c), optimal LIMA graft (d), and sequential bypass grafting (e) configurations. (b) Comparison of pressure distribution among the healthy $(a)$, stenosed (b), and single graft revascularization (c), optimal LIMA graft (d) and sequential bypass grafting (e) configurations.

TABLE 3. Coronary flow $(\mathrm{mL})$ within the first level branches of coronary tree for the healthy and stenosed coronary arteries, the complete revascularization with single and sequential grafting configurations obtained from 3D CFD simulations.

\begin{tabular}{|c|c|c|c|c|}
\hline CA branches & Healthy CA & Stenosed CA & Single graft revascularization ${ }^{a}$ & Sequential graft LIMA-OM \\
\hline Inno. A & 554 & $479(-13)$ & $477(-14)$ & $475(-14)$ \\
\hline LCCA & 125 & $129(4)$ & $128(3)$ & $128(3)$ \\
\hline VA & 62 & $71(14)$ & 70 (13) & $70(13)$ \\
\hline LSA & 93 & $107(15)$ & $106(14)$ & $106(14)$ \\
\hline Total $\mathrm{H}-\mathrm{N}$ & 833 & $786(-6)$ & $782(-6)$ & $779(-7)$ \\
\hline s1 & 13 & $12(-3)$ & $12(-4)$ & $12(-4)$ \\
\hline LAD_main & 32 & $28(-13)$ & $55(69)$ & $33(3)$ \\
\hline Diagonal & 20 & $17(-12)$ & $17(-13)$ & $21(4)$ \\
\hline Total LAD & 65 & $58(-11)$ & $84(30)$ & $66(2)$ \\
\hline RM & 27 & $29(5)$ & $28(3)$ & $28(4)$ \\
\hline LCX_main & 36 & $31(-14)$ & $37(2)$ & $37(2)$ \\
\hline OM & 31 & $27(-14)$ & $32(2)$ & $32(2)$ \\
\hline $\mathrm{m} 1$ & 21 & $18(-15)$ & 21 (1) & 21 (1) \\
\hline Total LCX & 88 & $76(-14)$ & $90(2)$ & $90(2)$ \\
\hline RCA & 77 & $59(-24)$ & 79 (3) & $80(3)$ \\
\hline DAo & 3955 & 4039 (2) & 3984 (1) & 4004 (1) \\
\hline
\end{tabular}

Acute flow adjustments in coronary flow are presented as percent variation (\%) from the healthy baseline anatomy is given in the parenthesis. ${ }^{a}$ After LIMA graft optimization coronary perfusion remained very similar ( $<2 \%$ variation) to the single graft revascularization level and these results are not presented in this table.

significantly higher than variation in LIMA graft resistance post-optimization. It is worthwhile to note that single LIMA grafting in both original and optimum designs allowed higher LAD flow $(\sim 30 \%)$ compared to both sequential graft and healthy baseline. As such, the pressure drop across the LAD branches was relatively higher in both original and optimal single LIMA grafts. 

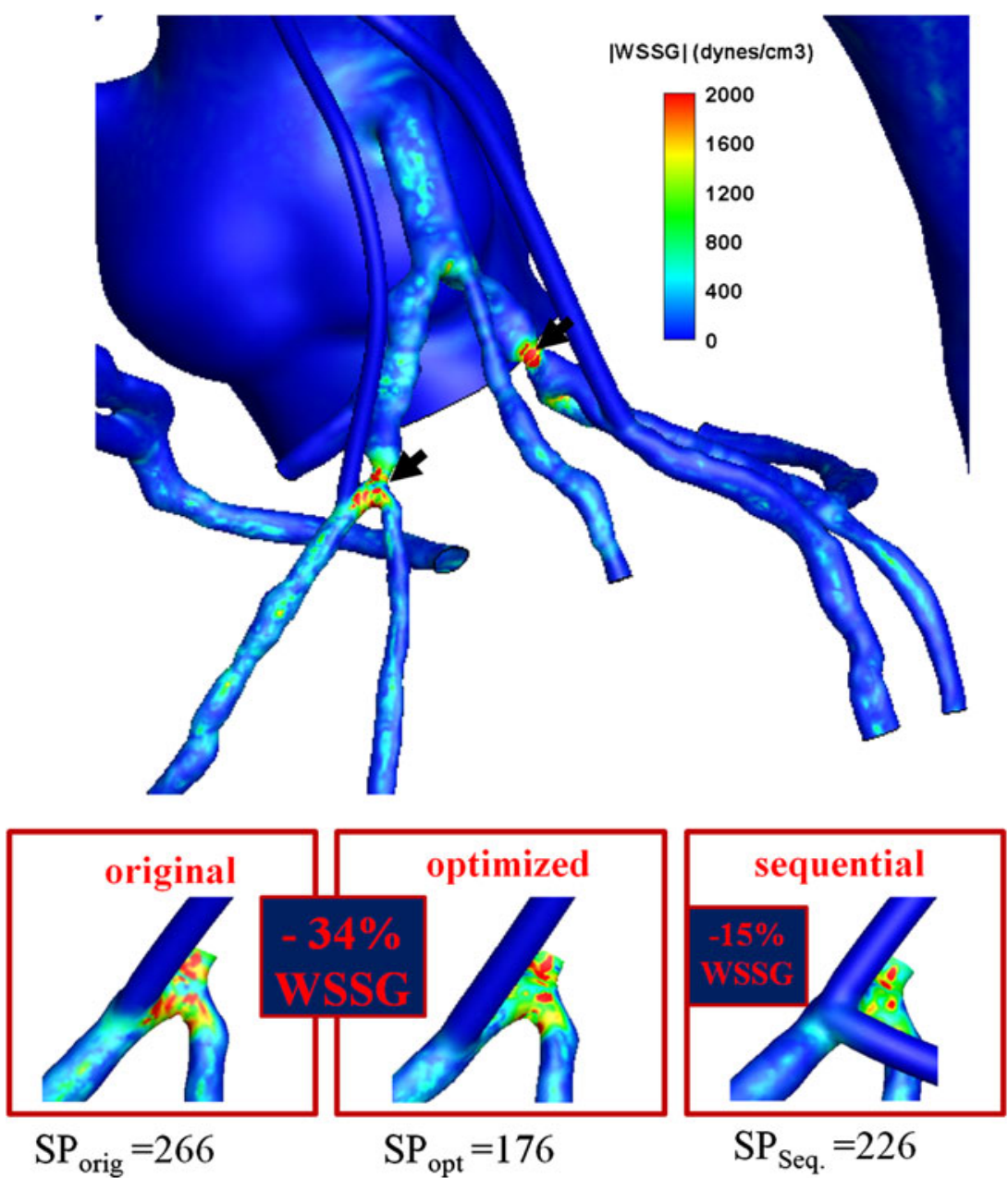

FIGURE 7. Spatial variation of wall shear stress (WSS) is quantified using WSS gradient (WSSG) (top). Elevated WSSG was found in proximity to the bifurcations and anastomosis zones (marked with black arrows), which are prone to intimal hyperplasia and atherosclerosis development. ${ }^{24} 3 \mathrm{D}$ evaluation of the optimized LIMA graft indicated significant WSSG relief $(\sim 34 \%)$ in agreement with the 2D simulations (bottom). WSSG was lowered by 15\% proximal to LAD and diagonal bifurcation for the sequential graft in comparison to the original graft.

WSSG was elevated significantly in proximity of the bifurcations and anastomosis zones, where the flow mixing from multiple branches alters unidirectional flow pattern as shown in Fig. 7. 3D evaluation of the optimized LIMA graft indicated significant WSSG relief $(-34 \%)$ in agreement with the 2D simulations. In comparison to the original LIMA grafts, WSSG was lowered by $15 \%$ proximal to LAD and diagonal bifurcation of sequential graft. Hence, LAD-LCX sequential graft provided improved hemodynamics as an alternative to the single LAD and LCX bypasses.

Coronary flow along the first level CA tree manifested a non-Newtonian blood rheology according to Ballyk blood rheology model (Fig. 8). $I_{\mathrm{L}}$ was elevated along the torturous sections of RCA, dilations and bifurcations within the left coronary artery tree, where the strain rate was reduced considerably. Carreau model produced relatively higher $I_{\mathrm{L}}$ due to the

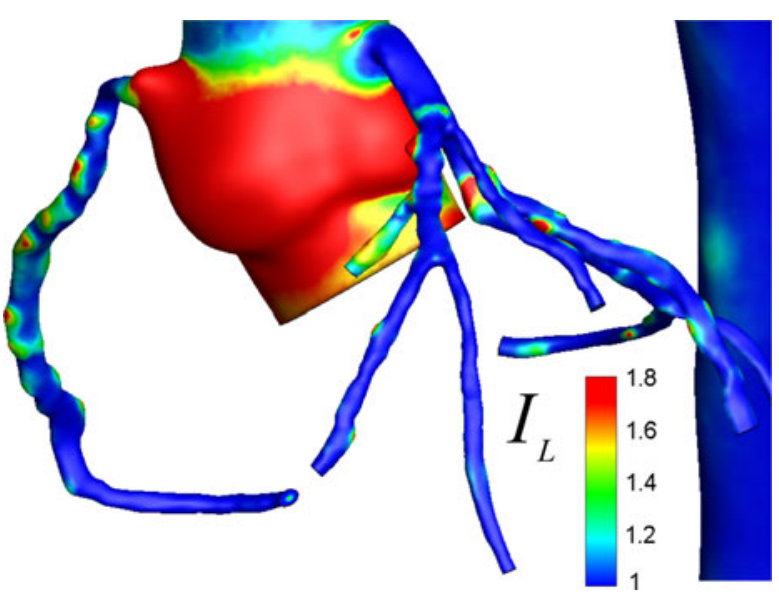

FIGURE 8. Distribution of non-Newtonian importance factor, $I_{L}$ is plotted on the coronary artery model (based on Ballyk model). Shear-thinning rheology of the coronary flow was significant along the modeled coronary artery tree (i.e., from the sinus to the end of the first generation branches). 
well-known under-estimation of the blood viscosity in low shear zones. ${ }^{21}$

\section{DISCUSSION}

For the fixed distal and proximal anastomosis sites, i.e., not necessarily the anastomosis angles, multi-scale CFD coupled shape optimization was used to investigate the optimal in vivo shape of the CABGs. Preceding the $2 \mathrm{D}$ formal shape optimization, 3D patientspecific CFD simulations validated the utility of the proposed 2D CABG optimization method and evaluated the hemodynamic performance of the selected CABG configurations. Starting the surgical design process with reduced order $2 \mathrm{D}$ optimization allowed a narrower the search space and a computationally efficient framework, which provided fast evaluation of initial design alternatives. Optimization results for the LIMA graft indicated that the WSSG at the anastomosis zone can be reduced by $\sim 30 \%$ between suboptimal and optimal configurations. Clinically, the lower WSSG may translate to improved hemodynamic performance; in turn, it will provide higher patency rates to prevent postoperative graft restenosis. We demonstrated that hemodynamics efficiency of the LIMA graft depended not only on the anastomosis angle but also on the vessel curvature for the fixed anastomosis angle. Therefore, for the first time in literature, this study identified the importance of the bulk shape of the CABG, which has been overlooked previously. In addition, high WSSG identified proximal to CA bifurcations may provide a guideline for selecting the distal anastomosis site to prevent postoperative graft restenosis.

According to our patient-specific 3D CFD analysis, sequential grafting improved the local hemodynamics proximal to LAD and diagonal bifurcation by lowering the local WSSG and WSS in comparison to original single LIMA grafting. These results promise higher potency for the sequential grafting method and agree with previous results based on idealized models ${ }^{45}$ and the long term follow up studies favoring the performance of composite grafting over single grafts. ${ }^{7,8,52}$ As opposed to the clinical data ${ }^{30}$ and results based on circuit-analog lumped models of coronary circulation, ${ }^{38}$ sequential grafting failed to increase proximal graft (LIMA) flow. For the present 3D model, the distal end of the sequential graft was anastomosed approximately at a similar peripheral distance from the coronary sinus in comparison to the distal anastomosis site of LIMA graft. Hence, the downstream pressure at the distal end of the sequential graft, which affects the graft flow and patency, ${ }^{29}$ was similar to downstream pressure at the distal end of the LIMA graft. Future clinical and numerical studies should investigate the effect of peripheral anastomosis of the distal end of the sequential graft to improve the proximal graft flow.

Results based on two common blood rheology models indicated that shear-thinning behavior of the blood flow was significant along the modeled coronary artery tree (i.e., from the sinus to the end of the first generation branches). Hence, our findings suggest that analysis and optimization of the coronary flow conduits requires incorporating non-Newtonian blood rheology.

Various cost functions employed in this study highlighted the necessity for reliable case-specific cost functions for hemodynamics optimization problems confirming the earlier cardiovascular CFD-coupled optimal shape studies. ${ }^{25}$ Shape optimization based on energy efficiency indices and $\Phi$ resulted in small curvature topology, indicating that tortuosity of the CABG effects the rotational (dissipative) characteristics of blood flow. We suspect that the influence of $\Phi$ will be more prevalent in the presence of transient flow regimes and the retrograde flow borne flow structures. Future optimization studies under pulsatile coronary flow settings should investigate the importance of oscillating shear index and flow recirculation at the anastomosis site. Oscillating shear index has been correlated strongly with vasoregulation and disease states. ${ }^{24}$ The current optimization paradigm incorporated the anatomical constraints (pulmonary vasculature, location of aorta, and heart) based on a single 2D CT slice. Under transient flow conditions, an optimization paradigm incorporating a large design space, to account for the optimal CABG design based on $3 \mathrm{D}$ anatomical constraints is needed, but requires high performance computing resources. ${ }^{32}$

It is worthwhile to note that results presented in this study were based solely on a single clinical case with healthy aorta-coronary tree architecture. Therefore, the proposed methodology needs to be expanded on a larger patient cohort in order to address the complexity which could arise in a broader spectrum of CABG geometries, i.e., abnormal coronary tree, congenital coronary defects, flow competition between neighboring CABGs in comparison to the hemodynamics performance of a single graft. Likewise, the stenosis severity on each main coronary artery was modeled at a fixed clinically moderate level irrespective of the actual pathology of the patient's coronary tree. Future studies will identify the effect of stenosis severity on the performance and optimal shape of the graft in order to validate the proposed pre-surgical planning paradigm. In addition, the post-op in vivo CABG exhibits dynamic conformational variations due to cardiac contractions. Therefore, the results presented in this manuscript may also demonstrate the 
extent of vascular resistance variation under physiological conditions. Shape optimization under dynamic myocardial loading conditions requires further investigation.

The CFD-coupled shape optimization framework evaluated in this study allowed fast convergence due to the efficient FEMLAB 2D solver and robust communication between the optimization toolbox and FEA package, both built in MATLAB. Particularly, the third-order Bezier curves allowed easy geometry modulation and reproduced the 2D graft shape accurately under the consideration of in vivo geometrical constrains. This shows promise for extending this methodology to optimize complex connections used in reconstructive surgeries for congenital heart defects and for the future surgical planning challenges. The design paradigm developed here could be expanded to other surgical connections such as the shunts used in reconstructive surgeries for single ventricles (i.e., Norwood Procedure) or femoral arteries (femoropoptiteal bypass), etc. Recently, we embarked upon translating the CFD-guided optimal surgical design concept to the growth and remodeling of embryonic aortic arches, which represent one of the most complex components in the cardiovascular system. ${ }^{55}$ These studies highlight the prospective use of the CFDcoupled shape optimization approach in cardiovascular research and point to future directions for improving understanding of optimal surgical design and cardiovascular function.

\section{CONCLUSIONS}

An automated framework for coupling optimal shape design to non-Newtonian blood flow simulation in multi-scale patient-specific cardiovascular geometries is demonstrated. The proposed sketch-based surgical planning paradigm evaluated the selected coronary bypass surgery procedures based on local hemodynamics and acute hemodynamic readjustments of aorta-CA flow. Our results indicated lower local WSSG for both the optimized LIMA graft and the LAD-LCX sequential graft in comparison to the original LIMA graft. This procedure may provide a rational to aid surgical decision-making process in time-critical, patient-specific CA bypass operations before the in vivo execution. We showed that the SQP optimization method realizes robust convergence provided the proper cost function and initial condition are selected. Optimal shape design requires evaluating the relation between the cost functions and flow regions, which would cause CABG failure. It is also critical to understand the behavior of each cost function as it pertains to design quality. Future studies should benefit from multi-objective optimization to minimize local flow disturbances (WSSG) and flow rotationality $(\Phi)$, and to maximize the conduit energy efficiency, concurrently. Specifically, it is required to optimize the anastomosis geometry as well as the graft length and transitional curvature to achieve hemodynamic characteristics that promote failure-free bypass conduits.

\section{ACKNOWLEDGMENTS}

The study was partially supported through NSF CAREER 0954465 and Pennsylvania Infrastructure Technology Alliance (PITA). The computational resources provided in part by Pittsburgh Supercomputing Center grant CCR080013. The authors would like to thank Gunay Orbay, MS for his valuable contributions in implementing the sketch-based anatomical shape editing progress.

\section{REFERENCES}

${ }^{1}$ Abraham, F., et al. Shape optimization in steady blood flow: a numerical study of non-Newtonian effects. Comput. Methods Biomech. Biomed. Eng. 8:127-137, 2005.

${ }^{2}$ Agoshkov, V., et al. A mathematical approach in the design of arterial bypass using unsteady stokes equations. $J$. Sci. Comput. 28:139-165, 2006.

${ }^{3}$ Brien, T. O., et al. On reducing abnormal hemodynamics in the femoral end-to-side anastomosis: the influence of mechanical factors. Ann. Biomed. Eng. 33:310-322, 2005.

${ }^{4}$ Caiati, C., et al. New noninvasive method for coronary flow reserve assessment: contrast-enhanced transthoracic second harmonic echo Doppler. Circulation 99:771-778, 1999.

${ }^{5}$ Canver, C. C. Conduit options in coronary artery bypass surgery. Chest 108:1150-1155, 1995.

${ }^{6}$ Chatzizisis, Y. S., et al. Role of endothelial shear stress in the natural history of coronary atherosclerosis and vascular remodeling: molecular, cellular, and vascular behavior. J. Am. Coll. Cardiol. 49:2379-2393, 2007.

${ }^{7}$ Christenson, J. T., and M. Schmuziger. Sequential venous bypass grafts: results 10 years later. Ann. Thorac. Surg. 63:371-376, 1997.

${ }^{8}$ Dion, R., et al. Complementary saphenous grafting: longterm follow-up. J. Thorac. Cardiovasc. Surg. 122:296-304, 2001.

${ }^{9}$ Dur, O., et al. Optimization of inflow waveform phasedifference for minimized total cavopulmonary power loss. J. Biomech. Eng. 132:031012-(9), 2010.

${ }^{10}$ Edelman, R. R., et al. Flow velocity quantification in human coronary arteries with fast, breath-hold MR angiography. J. Magn. Reson. Imaging. 3:699-703, 1993.

${ }^{11}$ Fei, D. Y., et al. The effect of angle and flow rate upon hemodynamics in distal vascular graft anastomoses: a numerical model study. J. Biomech. Eng. 116:331-336, 1994.

${ }^{12}$ Frakes, D. H., et al. New techniques for the reconstruction of complex vascular anatomies from MRI images. J. Cardiovasc. Magn. Reson. 7:425-432, 2005. 
${ }^{13}$ Gibson, C. M., et al. Relation of vessel wall shear stress to atherosclerosis progression in human coronary arteries. Arterioscler. Thromb. 13:310-315, 1993.

${ }^{14}$ Grundy, S. M., et al. Diabetes and cardiovascular disease: a statement for healthcare professionals from the American Heart Association. Circulation 100:1134-1146, 1999.

${ }^{15}$ Hund, S. Hemodynamic Design Optimization of a Ventricular Cannula: Evaluation and Implementations of Objective Functions. MS Thesis, Biomedical Engineering, UPitt, Pittsburgh, 2006.

${ }^{16}$ Kara, L. B., and K. Shimada, Construction and modification of 3D geometry using a sketch-based interface. Presented at the EUROGRAPHICS Workshop on SketchBased Interfaces and Modeling, 2006.

${ }^{17}$ Kara, L. B., and K. Shimada. Sketch-based 3D shape creation for industrial styling design. IEEE Comput. Graph. Appl. 27:554-567, 2007.

${ }^{18}$ Kara, L. B., et al. Pen-based styling design of 3D geometry using concept sketches and template models. Presented at the ACM Solid and Physical Modeling Conference, 2006.

${ }^{19}$ Kasik, D., et al. Ten CAD challenges. IEEE Comput. Graph. Appl. 25:84-92, 2005.

${ }^{20}$ Kute, S. M., and D. A. Vorp. The effect of proximal artery flow on the hemodynamics at the distal anastomosis of a vascular bypass graft: computational study. J. Biomech. Eng. 123:277-283, 2001.

${ }^{21}$ Lee, S. W., and D. A. Steinman. On the relative importance of rheology for image-based CFD models of the carotid bifurcation. J. Biomech. Eng. 129:273-278, 2007.

${ }^{22}$ Lei, M., et al. Geometric design improvements for femoral graft-artery junctions mitigating restenosis. J. Biomech. 29:1605-1614, 1996.

${ }^{23}$ Lei, M., et al. Computational design of a bypass graft that minimizes wall shear stress gradients in the region of the distal anastomosis. J. Vasc. Surg. 25:637-646, 1997.

${ }^{24}$ Loth, F., et al. Relative contribution of wall shear stress and injury in experimental intimal thickening at PTFE endto-side arterial anastomoses. J. Biomech. Eng. 124:44-51, 2002.

${ }^{25}$ Marsden, A., et al. A computational framework for derivative-free optimization of cardiovascular geometries. Comput. Methods Appl. Mech. Eng. 197:1890-1905, 2008.

${ }^{26}$ Marsden, A. L., et al. Evaluation of a novel Y-shaped extracardiac Fontan baffle using computational fluid dynamics. J. Thorac. Cardiovasc. Surg. 137:394-403, 2009.

${ }^{27}$ Migliavacca, F., et al. Computational fluid dynamics simulations in realistic 3-D geometries of the total cavopulmonary anastomosis: the influence of the inferior caval anastomosis. J. Biomech. Eng. 125:805-813, 2003.

${ }^{28}$ Myers, J. G., et al. Factors influencing blood flow patterns in the human right coronary artery. Ann. Biomed. Eng. 29:109-120, 2001 .

${ }^{29}$ Nishida, H., et al. Flow study of surgical coronary artery fistula as an alternative to sequential bypass. Cardiovasc. Surg. 3:375-380, 1995.

${ }^{30} \mathrm{O}$ 'Neill, Jr., M. J., et al. A rationale for the use of sequential coronary artery bypass grafts. J. Thorac. Cardiovasc. Surg. 81:686-690, 1981.

${ }^{31}$ Papaharilaou, Y., et al. The influence of out-of-plane geometry on pulsatile flow within a distal end-to-side anastomosis. J. Biomech. 35:1225-1239, 2002.

${ }^{32}$ Payli, R., et al. High performance clinical computing on the TeraGrid: patient-specific hemodynamic analysis and surgical planning. TeraGrid 2007 Conference, Madison, WI, 2007.
${ }^{33}$ Pekkan, K., et al. Total cavopulmonary connection flow with functional left pulmonary artery stenosis: angioplasty and fenestration in vitro. Circulation 112:3264 3271, 2005.

${ }^{34}$ Pekkan, K., et al. Physics-driven CFD modeling of complex anatomical cardiovascular flows-a TCPC case study. Ann. Biomed. Eng. 33:284-300, 2005.

${ }^{35}$ Pekkan, K., et al. Neonatal aortic arch hemodynamics and perfusion during cardiopulmonary bypass. J. Biomech. Eng. 130:061012, 2008.

${ }^{36}$ Pekkan, K., et al. Patient-specific surgical planning and hemodynamic computational fluid dynamics optimization through free-form haptic anatomy editing tool (SURGEM). Med. Biol. Eng. Comput. 46:1139-1152, 2008.

${ }^{37}$ Pekkan, K., et al. Hemodynamic performance of stage-2 univentricular reconstruction: Glenn vs. hemi-Fontan templates. Ann. Biomed. Eng. 37:50-63, 2009.

${ }^{38}$ Pietrabissa, R., et al. A lumped parameter model to evaluate the fluid dynamics of different coronary bypasses. Med. Eng. Phys. 18:477-484, 1996.

${ }^{39}$ Quarteroni, A., and G. Rozza. Optimal control and shape optimization of aorto-coronaric bypass anastomoses. Math. Model Methods Appl. Sci. 13:1801-1823, 2003.

${ }^{40}$ Raghavan, M. L., et al. Regional distribution of wall thickness and failure properties of human abdominal aortic aneurysm. J. Biomech. 39:3010-3016, 2006.

${ }^{41}$ Raja, S. G. Composite arterial grafting. Expert Rev. Cardiovasc. Ther. 4:523-533, 2006.

${ }^{42}$ Rosamond, W., et al. Heart disease and stroke statistics-2008 update: a report from the American Heart Association Statistics Committee and Stroke Statistics Subcommittee. Circulation 117:e25-e146, 2008.

${ }^{43}$ Rozza, G. On optimization, control and shape design of an arterial bypass. Int. J. Numer. Methods Fluids 47:1411-1419, 2005.

${ }^{44} \mathrm{Ryu}, \mathrm{K}$., et al. Importance of accurate geometry in the study of the total cavopulmonary connection: computational simulations and in vitro experiments. Ann. Biomed. Eng. 29:844-853, 2001.

${ }^{45}$ Sankaranarayanan, M., et al. Blood flow in an out-of-plane aorto-left coronary sequential bypass graft. Comput. Cardiovasc. Mech. 2:277-295, 2010.

${ }^{46}$ Schittowski, K. NLQPL: a FORTRAN-subroutine solving constrained nonlinear programming problems. Ann. Oper. Res. 5:485-500, 1985.

${ }^{47}$ Sherwin, S. J., et al. The influence of out-of-plane geometry on the flow within a distal end-to-side anastomosis. $J$. Biomech. Eng. 122:86-95, 2000.

${ }^{48}$ Soulis, J. V., et al. Non-Newtonian models for molecular viscosity and wall shear stress in a $3 \mathrm{D}$ reconstructed human left coronary artery. Med. Eng. Phys. 30:9-19, 2008.

${ }^{49}$ Sundareswaran, K. S., et al. Correction of pulmonary arteriovenous malformation using image-based surgical planning. JACC Cardiovasc. Imaging 2:1024-1030, 2009.

${ }^{50}$ Taylor, C. A., and C. A. Figueroa. Patient-specific modeling of cardiovascular mechanics. Annu. Rev. Biomed. Eng. 11:109-134, 2009.

${ }^{51}$ Torii, R., et al. A computational study on the influence of catheter-delivered intravascular probes on blood flow in a coronary artery model. J. Biomech. 40:2501-2509, 2007.

${ }^{52}$ Vural, K. M., et al. Long-term patency of sequential and individual saphenous vein coronary bypass grafts. Eur. J. Cardiothorac. Surg. 19:140-144, 2001. 
${ }^{53}$ Walsh, M. T., et al. On the existence of an optimum endto-side junctional geometry in peripheral bypass surgerya computer generated study. Eur. J. Vasc. Endovasc. Surg. 26:649-656, 2003.

${ }^{54}$ Wang, C., et al. Progress in the CFD modeling of flow instabilities in anatomical total cavopulmonary connections. Ann. Biomed. Eng. 35:1840-1856, 2007.

${ }^{55}$ Wang, Y., et al. Aortic arch morphogenesis and flow modeling in the chick embryo. Ann. Biomed. Eng. 37:1069-1081, 2009.
${ }^{56}$ Wieneke, H., et al. Determinants of coronary blood flow in humans: quantification by intracoronary Doppler and ultrasound. J. Appl. Physiol. 98:1076-1082, 2005.

${ }^{57}$ Wootton, D. M., and D. N. Ku. Fluid mechanics of vascular systems, diseases, and thrombosis. Annu. Rev. Biomed. Eng. 1:299-329, 1999.

${ }^{58}$ Zarins, C. K., and C. A. Taylor. Endovascular device design in the future: transformation from trial and error to computational design. J. Endovasc. Ther. 16(Suppl 1):I12I21, 2009. 